\title{
N-(3-Oxododecanoyl) Homoserine Lactone Is a Generalizable Plasma Membrane Lipid-Ordered Domain Modifier
}

\begin{abstract}
Hefei Ruan ${ }^{1 \dagger}$, Chunlin Zou ${ }^{1 \dagger}$, Yanni Xu ${ }^{1 \dagger}$, Xiaohong Fang ${ }^{2,3}$, Tie Xia ${ }^{1 *}$ and Yan Shi ${ }^{1,4 *}$
${ }^{1}$ Beijing Key Lab for Immunological Research on Chronic Diseases, Department of Basic Medical Sciences, Tsinghua-Peking University Joint Center for Life Sciences, School of Medicine, Institute for Immunology, Tsinghua University, Beijing, China, ${ }^{2}$ Key Laboratory of Molecular Nanostructure and Nanotechnology, CAS Research/Education Center for Excellence in Molecular Sciences, Institute of Chemistry, Chinese Academy of Sciences, Beijing, China, ${ }^{3}$ University of Chinese Academy of Sciences, Beijing, China, ${ }^{4}$ Department of Microbiology, Immunology and Infectious Diseases, Snyder Institute, University of Calgary, Calgary, $A B$, Canada
\end{abstract}

\section{OPEN ACCESS}

Edited by:

Patricia Marie Kane,

Upstate Medical University,

United States

Reviewed by:

Oskar Engberg,

University Hospital Leipzig, Germany

Bidisha Sinha,

Indian Institute of Science Education

and Research Kolkata, India

*Correspondence:

Tie Xia

xiatie@mail.tsinghua.edu.cn

Yan Shi

yanshiemail@mail.tsinghua.edu.cn

†These authors have contributed equally to this work

Specialty section: This article was submitted to

Membrane Physiology

and Membrane Biophysics,

a section of the journal

Frontiers in Physiology

Received: 14 August 2021 Accepted: 28 December 2021

Published: 22 February 2022

Citation:

Ruan H, Zou C, Xu Y, Fang X,

Xia T and Shi Y (2022)

N-(3-Oxododecanoyl) Homoserine Lactone Is a Generalizable Plasma

Membrane Lipid-Ordered Domain

Modifier. Front. Physiol. 12:758458.

doi: 10.3389/fphys.2021.758458
A mammalian plasma membrane is a structure on which several layers of complexity are built. The first order of complexity comes from the heterogeneity of lipid-ordered domains. Gangliosides in concert with cholesterol are preferentially packed on the outer leaflet and form lipid-ordered domains, commonly known as lipid rafts. The formation and dynamics of these domains impact nearly all membrane protein functions and are an intensely studied topic. However, tools suited for lipid domain alteration are extremely limited. Currently, methyl- $\beta$-cyclodextrin (M $\beta C D$ ) appears to be the most common way to disrupt lipid domains, which is believed to operate via cholesterol extraction. This significantly limits our ability in membrane biophysics research. Previously, we found that $\mathrm{N}$-(3-oxo-dodecanoyl) homoserine lactone (3oc), a small signaling chemical produced by Pseudomonas aeruginosa, is highly efficient in altering lipid-ordered domains. In this study, 3oc was compared with M $\beta C D$ in a series of biochemical, biophysical, and cell biological analyses. Per molarity, 3oc is more efficient than $\mathrm{M} \beta C D$ in domain alteration and appears to better retain membrane lipids after treatment. This finding will provide an essential reagent in membrane biophysics research.

Keywords: lipid raft, 3oc, M $\beta C D$, plasma membrane, cholesterol, lipid-ordered domain

\section{INTRODUCTION}

The eukaryotic plasma membrane is heterogeneous in lipid composition as well as in protein contents. It is recognized that this heterogeneity is critical for transmembrane receptor signaling (Simons and Toomre, 2000; Shi and Ruan, 2020). While the complexity is multidimensional, lipidordered domains are considered to be at the root whereby other heterogeneities are further added. It is generally accepted that glycosylated gangliosides, particularly sphingomyelin, are preferentially packed on the outer leaflet with positive curvature. Coupled to the repulsion of polyunsaturated phospholipids, the cone-shaped gangliosides are stabilized by small cholesterol to form a vertically slightly elevated area known as lipid-ordered domain (Lo, also known as lipid rafts) in reference with the rest of the membrane ( $L d$ or lipid disordered domains) (García-Arribas et al., 2016; Wang et al., 2017). These domains are highly dynamic and are in constant transition of formation (demixing) and dissolution (mixing) (Kusumi et al., 2005). As they are under optical diffraction, 
indirect measurements have different estimations of their sizes, ranging from 20 to $200 \mathrm{~nm}$ (Pralle et al., 2000; Pike, 2006).

While the biochemical and biophysical characterization is ongoing, as proposed by Kai Simons, the role of lipid rafts in receptor signaling regulation has been confirmed in essentially all aspects of mammalian biology, particularly immunology, development, cancer, and neurology (Lee et al., 2010; Asakura et al., 2015; Nakayama et al., 2018; Vona et al., 2021). For instance, the components of the T cell receptor (TCR) complex are distributed either in or out of $L o$ at the resting state. TCR signaling triggers a reorganization of those components to form a large disk-like structure driven by lipid dynamics (Orbach and Su, 2020; Zhang et al., 2021). Lo domains also sort the lipid species on the inner leaflet which leads to the regulated exposure of secondary messengers and stabilizes the plasma membrane onto the cortical cytoskeleton (Mu et al., 2018).

One of the commonly used approaches in earlier years was to extract lipid rafts with cold non-ionic detergents (i.e., Triton X100), which resulted in detergent-resistant membrane domains (DRMs). However, DRMs are increasingly regarded as imprecise representations of real-life lipid domains (Levental et al., 2020). Another approach is to extract cholesterol from the plasma membrane. Cyclodextrins are cyclic oligosaccharides that contain a hydrophobic core, with a hydrophilic outer surface. Their water solubility and affinity to membrane permit their use as a tool to extract cholesterol from the plasma membrane (Ohvo and Slotte, 1996; López et al., 2011). Cholesterol-saturated cyclodextrins can also be used as a shuttle to increase the cholesterol content in live-cell membranes. This technique has been the main staple in lipid domain research and is the technical basis for a lot of literature on lipid rafts. However, the technique is inefficient and time-consuming, requiring often more than one round of depletion. In our previous study, we found that the LasI-LasR circuit of quorum sensing in Pseudomonas aeruginosa relies on the production of $\mathrm{N}$-(3-oxo-dodecanoyl) homoserine lactone (3oc) (Song et al., 2019). 3oc is essential for P. aeruginosa to inhibit host immune attack. Mechanistically, 3oc inserts itself into the lipid-ordered domains of host immune cells and causes their dissolution. This leads to the spontaneous trimerization of tumor necrosis factor receptor 1 (TNFR1), resulting in apoptotic cell death in host immune cells. 3oc is highly efficient, with a working concentration of low $\mu \mathrm{M}$. In this report, we systematically compared 3oc to methyl- $\beta$-cyclodextrin $(\mathrm{M} \beta \mathrm{CD})$ as a generalizable Lo modifier. Results indicate that the $30 \mathrm{c}$ is comparable to $\mathrm{M} \beta \mathrm{CD}$ by several key parameters and with some beneficial characters and suits the needs for lipid domain research.

\section{MATERIALS AND METHODS}

\section{Cell Culture}

Hela and COS-1 cells were cultured in Dulbecco's Modified Eagle Medium (DMEM, Gibco), supplemented with 10\% fetal bovine serum (FBS, HyClone), $100 \mathrm{mg} \mathrm{ml}^{-1}$ streptomycin, and $100 \mathrm{U}$ $\mathrm{ml}^{-1}$ penicillin at $37^{\circ} \mathrm{C}$ in a $5 \% \mathrm{CO}_{2}$ atmosphere. THP-1 cells were cultured in RPMI-1640 medium (HyClone), supplemented with $10 \% \mathrm{FBS}, 100 \mathrm{U} \mathrm{ml}^{-1}$ penicillin, $100 \mathrm{mg} \mathrm{ml}^{-1}$ streptomycin, $10 \mathrm{mM}$ HEPES, and $50 \mu \mathrm{M} \beta$-mercaptoethanol at $37^{\circ} \mathrm{C}$ in a $5 \%$ $\mathrm{CO}_{2}$ atmosphere.

\section{Giant Plasma Membrane Vesicle Isolation and Staining}

Before giant plasma membrane vesicle (GPMV) isolation, live cells were incubated with DiD (1:1,000, Invitrogen, V22887), which is a lipophilic fluorescent dye located in the disordered domain, for $10 \mathrm{~min}$ at $37^{\circ} \mathrm{C}$, then washed three times with "GPMV buffer" (10 mM HEPES, $150 \mathrm{mM} \mathrm{NaCl,} 2 \mathrm{mM} \mathrm{CaCl}$, $\mathrm{pH} 7.4$ ), and then incubated at $37^{\circ} \mathrm{C}$ for about $60 \mathrm{~min}$ in GPMV buffer containing $25 \mathrm{mM}$ PFA and $2 \mathrm{mM}$ DTT to induce vesiculation (Levental et al., 2010). The supernatant was collected and incubated with $0,1,10,30,100,200,500 \mu \mathrm{M}$, and 1, 5, 10, $30 \mathrm{mM} M \beta C D$ or $0,1,5,10,30 \mu \mathrm{M} 3 \mathrm{oc}$, and then used for threedimensional (3D) Confocal imaging to analyze the distribution of ordered and disordered domains.

\section{Membrane Components Extraction}

Hela cells were used to analyze the effect of M $\beta C D$ (SigmaAldrich, C4555) and 3oc (Cayman, 10007895) on cell membrane components. For cholesterol extraction experiment, because the efficiency of inserting cholesterol into the cell membrane by itself is very low, in this study, we first formed a saturated solution of BODIPY-cholesterol $(10 \mu \mathrm{g} / \mathrm{ml}$, Avanti Polar Lipids, $810255 \mathrm{P})$ and $\mathrm{M} \beta \mathrm{CD}$ in DMEM and then used the M $\beta C D$ to load cholesterol onto the cell membrane for $30 \mathrm{~min}$. After the cells were washed two times with DMEM, they were treated two times with $10 \mathrm{mM} \mathrm{M} \beta \mathrm{CD}$ or $10 \mu \mathrm{M}$ 3oc for $30 \mathrm{~min}$. For sphingomyelin (SM), phosphatidylcholine (PC), and phosphatidylethanolamine (PE) extraction experiment, fluorescent-labeled lipids NBD-SM (10 $\mu \mathrm{g} / \mathrm{ml}$, Avanti Polar Lipids, 810218P), TMR-PC (10 $\mu \mathrm{g} / \mathrm{ml}$, Avanti Polar Lipids, 810180P), and TMR-PE (10 $\mu \mathrm{g} / \mathrm{ml}$, Avanti Polar Lipids, 810241P) were used to label the cell membrane for $30 \mathrm{~min}$, after the cells were washed two times with DMEM and then treated with $10 \mathrm{mM} \mathrm{M} \beta C D$ or $10 \mu \mathrm{M} 3 \mathrm{oc}$ for $30 \mathrm{~min}$. Finally, these samples were fixed and then used for total internal reflection fluorescence microscope (TIRFM) imaging to analyze the corresponding lipid composition changes on the cell membrane.

\section{Analysis of Endogenous Lipid Changes by Liquid Chromatography-Mass Spectrometry}

Liquid chromatography-mass spectrometry (LC-MS) was used to measure changes in endogenous lipid composition. Hela cells were seeded in a 10-cm cell culture dish for $24 \mathrm{~h}$; before treatments, the cells were washed two times with phosphatebuffered saline (PBS) to remove cell debris and dead cells and then treated with $10 \mathrm{mM}$ or $10 \mu \mathrm{M}$ 3oc (both $\mathrm{M} \beta \mathrm{CD}$ and 3oc were diluted with PBS) for $30 \mathrm{~min}$. Cell supernatants were extracted two times with a mixture of dichloromethane and methanol (volume ratio 2:1). After drying with nitrogen, they were reconstituted with a mixture of dichloromethane and isopropanol (volume ratio 1:4), and then corresponding 
internal standards were added for LC-MS analysis. Among them, the concentration of cholesterol is calculated using cholesterol standards, and other lipids are calculated using isotope-labeled internal standards 18:1 (d9) SM, 15:0-18:1 (d7) PC, and 15:018:1 (d7) PE (Avanti Polar Lipids, 791649, 791637, and 791638, respectively). For cholesterol, LC-MS was performed on the AB SCIEX QTRAP 4500 (United States) triple quadrupole mass spectrometer in SRM and positive ionization mode. The LC separation was run on an XBridge BEH C18 column. Methanol and isopropanol (8:2) containing $0.5 \mathrm{mM}$ amine acetate were as solvent $\mathrm{A}$, and water containing $0.5 \mathrm{mM}$ ammonium acetate as solvent B. The MS parameters are as follows: APCI ion source temperature $400^{\circ} \mathrm{C}$; air curtain: 30 psi; collision activated dissociation (CAD) gas settings: medium, ion spray voltage: 5,500 V; and ion gas 1: 40 psi; all data use Analyst 1.6.3 Software analysis, and the optimized cholesterol MRM acquisition mode is the dehydration peak $(\mathrm{M} / \mathrm{Z} 369.4>161.2$; $369.4>147.3$; $\mathrm{CE}=30 \mathrm{~V}$ ) to draw a standard curve of cholesterol to calculate the content of the sample. For other lipids, LC-MS was performed on the AB SCIEX QTRAP 5500 (United States) triple quadrupole mass spectrometer, in SRM and positive and negative ionization modes. The LC separation was performed on an XBridge $\mathrm{BEH}$ C8 column using 70\% acetonitrile aqueous solution containing $5 \mathrm{mM}$ ammonium acetate as solvent $\mathrm{A}$ and $5 \mathrm{mM}$ isopropanol as solvent $\mathrm{B}$. The MS parameters are as follows: ESI ion source temperature $500^{\circ} \mathrm{C}$; air curtain: 30 psi; CAD gas settings: medium; ion spray voltage: 5,500 V/-4,500 V; ion gases 1 and 2: 50 psi. The data acquisition software is AB SCIEX Analyst 1.7.1, and the data analysis software is SCIEX OS-MQ 1.6.1.

\section{Atomic Force Spectroscopy of Cell Membranes}

Force measurements were performed by using a commercial atomic force microscope (Cellhesion200 from JPK Instrument, Berlin), which was mounted on top of the inverted optical microscope (Zeiss Axio Observer D1). A soft cantilever (nominal spring constant: $0.011 \mathrm{~N} / \mathrm{m}$, HYDRA2R-100NG, ApppNano, United States) with no tip modification was used to touch and pull the cell membrane tethers. The applied force was $0.5 \mathrm{nN}$, and the contact time was $5 \mathrm{~s}$ in constant height mode. The pulling rate is $10 \mu \mathrm{m} / \mathrm{s}$. Cells were seeded on the glass substrate in the culture medium 1 day before the force measurements. Typically about 50 cells were probed in each condition, and each cell was probed 3 times. The force curves were analyzed with JPK data processing software, and the last tether breaking force $(\mathrm{Ft})$ was used to compute the apparent membrane tension ( $\mathrm{T}$ ) based on the following formula: $T=\frac{F_{t}^{2}}{8 \pi^{2} K}$, where $K$ is membrane bending stiffness and is about $3 \times 10^{-19} \mathrm{~N} / \mathrm{m}$ on average (Sheetz, 2001).

Membrane tension of Hela cells in resting state and on treatment of $30 c$ and $\mathrm{M} \beta \mathrm{CD}$, respectively, was computated from the tether force measurements according to the abovementioned formula. For $30 c$ and $\mathrm{M} \beta \mathrm{CD}$ experiments, force probing was started after the 10-min incubation. Each data point was from one force measurement, and for each cell, 3 measurements were performed, and about 50 cells were probed for each experimental condition.

\section{Endocytosis of TLR4}

The THP-1 cells were used to study the effect of MBCD and $30 c$ on lipid raft-dependent endocytosis of TLR4. THP-1 cells were first treated with $10 \mu \mathrm{M}$ Chlorpromazine Hydrochloride (CPZ, Sigma-Aldrich, C0982) for $24 \mathrm{~h}$ and then treated with $10 \mathrm{mM} \mathrm{M} \beta \mathrm{CD}$ for $30 \mathrm{~min}$ or $10 \mu \mathrm{M} 30 \mathrm{c}$ for $10 \mathrm{~min}$, and then $10 \mu \mathrm{g} / \mathrm{ml}$ LPS was used to induce the endocytosis of TLR4 for $30 \mathrm{~min}$. Finally, the cells were fixed and then stained with primary antibody of TLR4 (Abcam, ab22048) and secondary antibody of anti-mouse Alexa 488 (Invitrogen, A-11001) for flow cytometry analysis.

\section{Confocal Imaging}

The cortical cytoskeleton imaging experiments were performed on Hela cells. Hela cells were seeded in a $35-\mathrm{mm}$ confocal dish $24 \mathrm{~h}$ before experiments, and then the cells were treated with $10 \mathrm{mM} \mathrm{M} \beta \mathrm{CD}$ or $10 \mu \mathrm{M}$ 3oc for $0,10,30,60,120$, and $240 \mathrm{~min}$. After the cells were fixed, Phalloidin-Alexa Fluor 568 (1:200, Invitrogen, A12380) and DAPI $(1: 1,000)$ were used to stain the cytoskeleton and nucleus separately. Finally, the confocal imaging was performed, and Image J was used for the statistical analysis of average fluorescence intensity.

The 3D imaging of GPMVs was performed at room temperature by a commercial Spinning Disk Confocal Microscope (Andor Dragonfly, Oxford Instruments) equipped with a $100 \times 1.45$ NA objective. During imaging, DiD was excited by a $647-\mathrm{nm}$ laser, and the emission was collected by using a 660-700 $\mathrm{nm}$ band-pass filter. The 3D imaging was recorded with $2,048 \times 2,048$ pixels and with a step size of $200 \mathrm{~nm}$. The two-dimensional (2D) and Z-stack images were processed by ImageJ "Z project” plugin.

\section{Total Internal Reflection Fluorescence Microscope Imaging}

The abundance of cholesterol (Chol), SM, PC, and PE on the cell membrane was analyzed by a commercial TIRFM (Nikon TiE Inverted Microscope) equipped with a $100 \times 1.49$ NA objective and an Andor iXon DU897 EMCCD. During imaging, BODIPYcholesterol and NBD-SM were excited by a 488-nm laser, and the emission was collected by using a 500-550 $\mathrm{nm}$ band-pass filter. TMR-PC and TMR-PE were excited by a 561-nm laser, and the emission was collected by using a 580-620 $\mathrm{nm}$ band-pass filter. After the respective process, we imaged the fluorescence intensity of the bottom surface of each group of cells and then used the ImageJ program to perform statistical analysis (about 30 cells per group).

\section{Epidermal Growth Factor Receptor and Extracellular Signal-Regulated Kinase Activation}

The COS-1 cells were seeded in 6-well plates before treatment to $70 \%$ confluency, and the media were switched to serumfree DMEM. Then, COS-1 cells were incubated with $\mathrm{M} \beta \mathrm{CD}$ or $30 c$ that dissolved in serum-free DMEM for the indicated times at $37^{\circ} \mathrm{C}$. After treatment, cells were washed with cold PBS and then lysed with RIPA buffer (Beyotime; P0013B). Cell 
lysates were centrifuged at $12,000 \mathrm{rpm}, 4^{\circ} \mathrm{C}$ for $10 \mathrm{~min}$ in an Eppendorf centrifuge, and the supernatants were collected. After boiling in $5 \times$ SDS sample buffer for $10 \mathrm{~min}$, the supernatants were run on $10 \%$ acrylamide gel for $90 \mathrm{~min}$ at $90 \mathrm{~V}$ and then transferred to nitrocellulose filter membrane (PALL; 66485) at $260 \mathrm{~mA}$ for $90 \mathrm{~min}$. For immunoblotting, the nitrocellulose filter membrane was blocked with $5 \%$ milk in Tris-buffered saline Tween-20 (TBS-T). After incubation with a specific primary antibody in primary antibody dilution buffer (Beyotime; P0256) overnight at $4^{\circ} \mathrm{C}$, the membrane was washed in TBS-T for 4 times and each time for $5 \mathrm{~min}$. Horseradish peroxidase (HRP)-conjugated secondary antibodies were diluted in TBS-T and incubated for $1 \mathrm{~h}$ at room temperature. Then, positive immune reactive signals were detected by Amersham Imager 600 in the detection reagent (Yeasen; 36208ES76). The antibodies against epidermal growth factor receptor (EGFR, 4267), phosphorylated EGFR (pEGFR, 4407), extracellular signalregulated kinase (ERK, 9102), phosphorylated ERK (pERK, 9101), and HRP-conjugated anti-mouse (7076) or rabbit (7074) secondary antibody were from CST.

\section{Caspase Activation}

The THP- 1 cells were diluted to $0.75 \times 10^{6}$ cells $/ \mathrm{ml}$ and seeded in 6-well plates. Then, the cells were treated with $30 \mathrm{c}$ for the indicated times at $37^{\circ} \mathrm{C}$. After treatment, cells were collected and centrifuged at 3,000 rpm for $3 \mathrm{~min}$ at $4^{\circ} \mathrm{C}$, and then cell pellets were lysed. Cell lysates were applied to the Western blots as mentioned earlier, but samples were run on $12.5 \%$ acrylamide gel. Antibodies against Caspase 3 (9665), cleaved Caspase 3 (9664), and GAPDH (51332) were from CST.

\section{Apoptosis}

The THP-1 cell apoptosis was detected by the Annexin V/PI Apoptosis Detection Kit (4A Biotech; FXP023-100), following the user guide. In brief, cells were diluted to $0.75 \times 10^{6}$ cells $/ \mathrm{ml}$ and seeded in a 12-well plate and then stimulated with $30 \mathrm{c}$ for the indicated concentrations. Notably, $5 \mathrm{~h}$ later, cells were collected and stained with Annexin V-FITC and PI, and then the apoptotic effect was measured by flow cytometry. To normalize for spontaneous cell death, the percentage of live cells was calculated as follows: Live cell\% $=($ Annexin $\mathrm{V}$ negative cells in $\mathrm{M} \beta \mathrm{CD}$ or $30 \mathrm{c}$ treatment/total cell)/(Annexin $\mathrm{V}$ negative cells in DMSO treatment/total cells) $\times 100 \%$.

\section{RESULTS}

\section{3-Oxododecanoyl and M $\beta C D$ Treatments Induce Different Lipid-Ordered Domain Alterations}

As live-cell lipid-ordered domains are below optical diffraction, and their disruption causes a significant change in morphology and signaling transduction, we decided to use GPMVs to characterize the effects of M $B C D$ and $30 c$ treatments, as they contain lipid domains observable under the conventional microscope in the absence of cellular activities
(Baumgart et al., 2007). Using DiD to reveal disordered domains, Figure 1 shows GPMV from Hela cells displayed patch-like lipid-ordered domains without treatment, which are larger than those forming on the live-cell membrane (Pralle et al., 2000; Pike, 2006). From the $\mathrm{Z}$-stack, the $\mathrm{DiD}$ label-free areas were round, likely representing the gel phase in the presence of SM and cholesterol. M $\beta C D$ and 3oc treatments both removed those structures, in $2 \mathrm{D}$ view or in Z-stack. Interestingly, $\mathrm{M} \beta \mathrm{CD}$ and 3 oc treatments resulted in very distinct domain alterations. $\mathrm{M} \beta \mathrm{CD}$ produced a more homogeneous surface, likely due to the extraction of cholesterol leading to mixing (diffusion) of the lipophilic fluorescent dye DiD. Some "gel" phases with irregular surrounds remained, which might be a different type of domain occurring in the absence of cholesterol (Bagatolli, 2006). In contrast, 3oc produced a more limited disintegration with small DiD islands remaining (Figure 1A and Supplementary Movies 1-3). We categorized GPMV into three types: One, represented by the control treatment with $2-5$ continuous $\mathrm{DiD}$ phases per vesicle in a 2D image, was termed mixed ordered and disordered (MOD). The second, represented by $\mathrm{M} \beta \mathrm{CD}$ treatment, with total DiD labeling of the entire circumference, was termed uniform disordered (UD), and the third, represented by 3 oc treatment, with more than 5 distinct DiD label areas, was termed disintegrated small domains (DSDs). Figure 1B shows that in untreated GPMV, MOD dominated as expected. However, $\mathrm{M} \beta \mathrm{CD}$ and 3oc preferentially resulted in UD and DSD, respectively. We also compared the concentrations of $\mathrm{M} \beta \mathrm{CD}$ and $30 c$ required to achieve membrane alteration. 3oc showed an initial effective concentration around $5 \mu \mathrm{M}$ with DSD features more apparent in 10 and $30 \mu \mathrm{M}$. In contrast, $\mathrm{M} \beta \mathrm{CD}$ led to a UD-dominated feature around $100 \mu \mathrm{M}$. In no circumstance, were DSD and UD dominant with $\mathrm{M} \beta \mathrm{CD}$ and 3oc treatments, respectively (Supplementary Figure 1). From these results, it appeared that both $30 c$ and $\mathrm{M} \beta \mathrm{CD}$ alter lipid-ordered domains, but their mechanisms are different.

\section{3-Oxododecanoyl Disrupts Lipid-Ordered Domains Without Changing Plasma Membrane Components}

As $\mathrm{M} \beta \mathrm{CD}$ is known to extract cholesterol from the bilayer membrane, we labeled cells with fluorescent Chol, SM, PC, and $\mathrm{PE}$ and measured their abundance following the two different treatments with total internal reflection fluorescence (TIRF) imaging. M $\beta C D$ reduced cholesterol label, while $30 \mathrm{c}$ did not. Remarkably, despite the proposed cholesterol specificity of $\mathrm{M} \beta C D$, its treatment removed all four lipid species with nearly identical efficacy, while $30 c$ showed no such depletion (Figure 2A). Although the addition of labeled lipids into the membrane did not cause an observable increase in cell death (Supplementary Figure 2), this experiment was unlikely to completely mimic the real lipid dynamics, as all three labels, namely, BODIPY, NBD, and TMR, are of considerable molecular weights and are attached to targeted species at different positions. To avoid artifacts related to the labeling, we decided to use internally controlled LC-MS to measure all the relevant lipids. 

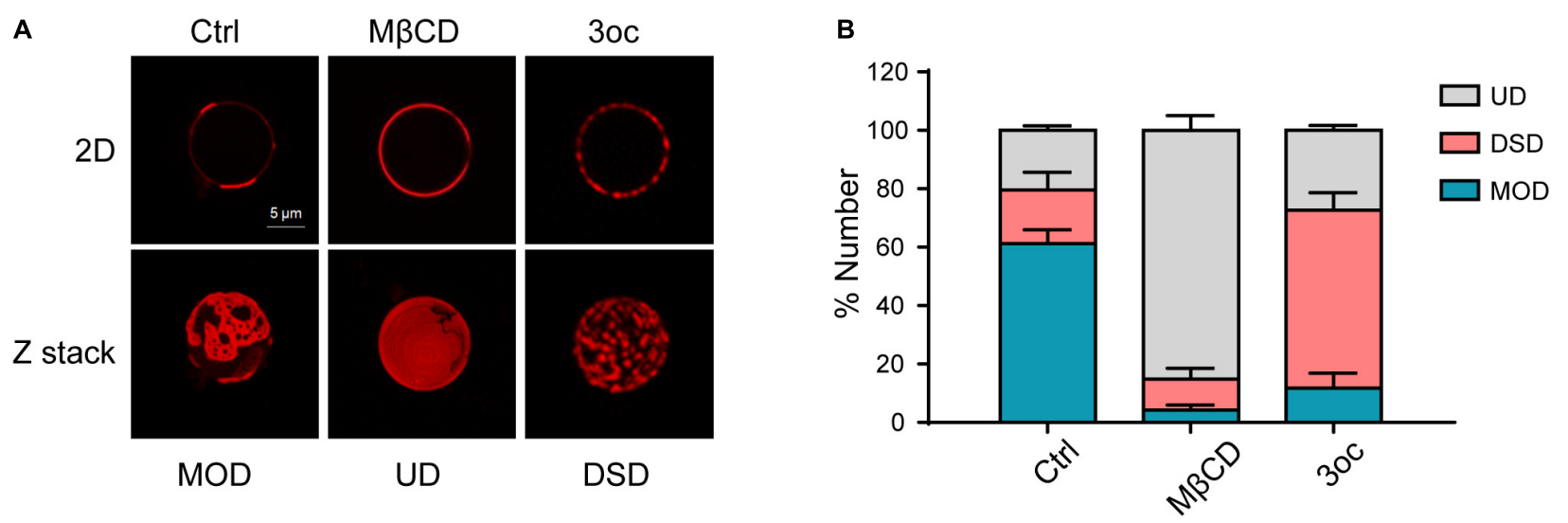

FIGURE 1 | Characterizing the effects of methyl- $\beta$-cyclodextrin (M $\beta C D)$ and $N$-(3-oxo-dodecanoyl) homoserine lactone (3oc) treatments on lipid-ordered domain on giant plasma membrane vesicles (GPMVs). (A) The upper panels show the distribution of ordered domains at the maximum diameter of GPMVs. Images from left to right are control, M $\beta C D(10 \mathrm{mM})$, and $30 \mathrm{c}(10 \mu \mathrm{M})$ treatments. The lower panels are the corresponding Z-stack images. (B) Statistical results show that mixed ordered and disordered (MOD) (59\%) dominates the untreated GPMVs, M $\beta C D$, and 3oc treatment preferentially resulted in uniform disordered (UD) (86.6\%) and disintegrated small domain (DSD) (63.05\%), respectively. This experiment was repeated three times, each with $40-50$ vesicles per condition. Scale bar $=5 \mu \mathrm{m}$.

Cholesterol LC absorption scan peak area was almost linear to sample amounts loaded, thus allowing the production of a standard curve. For treated samples, the amount of cholesterol present in the salutation phase was determined via curve fitting and it was found to be about 20-fold higher than the control while 3 oc did not show any increase (Figure 2B). Using isotopelabeled 18:1 (d9) SM, 15:0-18:1 (d7) PC, and 15:0-18:1 (d7) $\mathrm{PE}$ as internal ionization references, we performed LC-MS to measure the amounts of SM, PC, and PE of common acyl chain lengths ( 14-24) and saturation variants (Figure 2C). While these variants did not completely cover all possible acyl chain lengths of plasma membrane lipids, the general trend was clear that compared with $30 c, M \beta C D$ was uniquely capable of extracting all lipid species tested. This analysis of native lipid species gave a conclusion similar to Figure 2A. Regarding its cell biological impact, as plasma membrane lipid abundance is quantitatively maintained, $\mathrm{M} \beta \mathrm{CD}$ is expected to cause significant replenishment of membrane lipids, an effect spared by $30 c$. As accelerated plasma membrane lipid turnover alters the cell biological properties from several angles, particularly the lipid domain formation and signaling half-lives of surface receptors (Casares et al., 2019), we suggest additional caution in interpreting the cell signal change as $\mathrm{M} \beta \mathrm{CD}$ treatment results in more membrane behavior changes than what is associated with cholesterol extraction alone.

\section{3-Oxododecanoyl and M $\beta C D$ Treatments Lead to a Similar Reduction in Membrane Tension}

In our previous study, it was found that $30 \mathrm{c}$ causes a significant reduction in membrane packing (Song et al., 2019). This is expected to cause a reduction in tether force, an important property in cell or cell surface contact (Pontes et al., 2017). To quantify this alteration and compare the effects of the two treatments, we used atomic force microscopy (AFM) to analyze the force required to complete detachment following the treatments (Figure 3A; Sheetz, 2001). To avoid human bias, only the last step, i.e., tether breaking force, was selected (Sun et al., 2005; Li et al., 2020). Both M reduction in tether forces (Supplementary Figure 3), which can be translated into membrane tension per a published formula. In this study, both treatments led to a similar reduction in membrane tension (Figure 3B). While membrane tension can be a property of a pure bilayer, the extensive anchoring mechanisms between the inner leaflet and the cortical cytoskeleton contribute significantly to this physical property as well (Kapus and Janmey, 2013). To rule out this potential caveat, we treated cells with $10 \mu \mathrm{M} 30 \mathrm{oc}$ and $10 \mathrm{mM} \mathrm{M \beta CD}$ and chased the cortical skeletal change with Phalloidin. Overall, the impact on the F-actin intensity by both treatments was not profound, the intensity of staining waned slightly at 120 and $60 \mathrm{~min}$ for M $\mathrm{MCD}$ and 3oc, respectively (Supplementary Figure 4), and this was after the effect on the membrane tension started to appear ( $\sim 15 \mathrm{~min})$. In addition, if the force curves produced within the first $30 \mathrm{~min}$ after the treatment were compared with those produced in the next $30 \mathrm{~min}$, the overall reduction in tension by both treatments were similar (Supplementary Figure 5), suggesting that the tension change might not rely on the slow-onset cytoskeletal alteration. While we cannot rule out the indirect effect via cytoskeletal change induced by $\mathrm{M} \beta \mathrm{CD}$, our results seem to indicate that in the short-term treatments, both $\mathrm{M} \beta \mathrm{CD}$ and 3oc can reduce membrane tension.

\section{Lipid Raft Disruption Affects Cell Signal Transduction and Receptor Endocytosis}

As $\mathrm{M} \beta \mathrm{CD}$ and $30 \mathrm{oc}$ both disrupted lipid-ordered domains and reduced membrane tension to a similar extent, we wondered if they have similar effects in biological assays with regard to lipid raft disruption. One of the common effects of $\mathrm{M} \beta \mathrm{CD}$ cholesterol removal is the spontaneous activation of EGFR; we, therefore, 

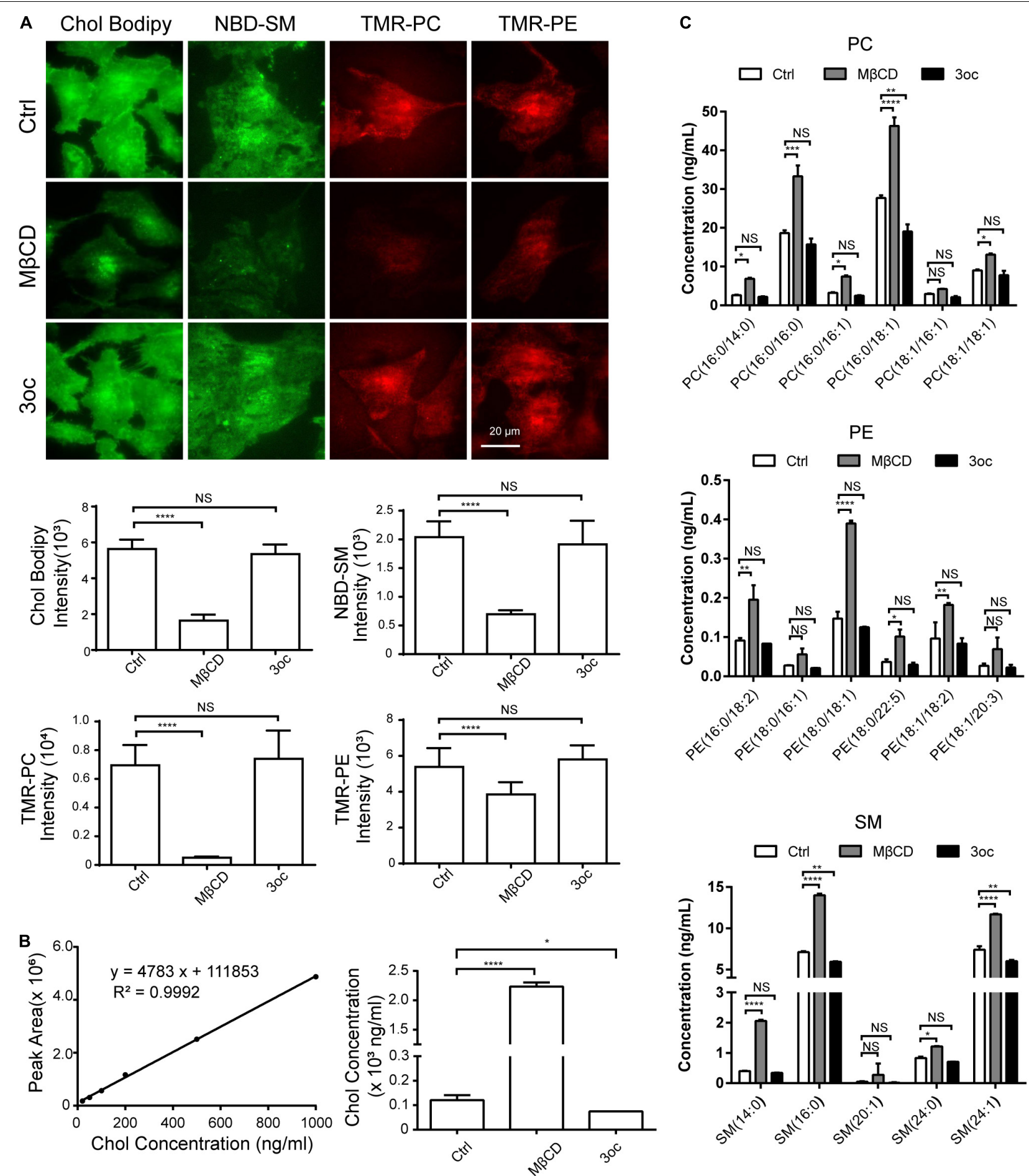

FIGURE 2 | Effects of M $\beta C D$ and 3oc treatments on cell membrane components. (A) Fluorescently labeled cholesterol (Chol), sphingomyelin (SM), phosphatidylcholine (PC), and phosphatidylethanolamine (PE) are used to analyze the effects of total internal reflection fluorescence microscope (TIRFM) imaging. From left to right, BODIPY-Chol, NBD-SM, TMR-PC, and TMR-PE are used to label the Hela cell membrane. The histograms below are the average fluorescence intensity of about 30 cells in each group. Significant differences were analyzed by an unpaired $t$-test. (B) The concentrations of endogenous cholesterol in cell supernatant after $\mathrm{M} \beta \mathrm{CD}$ and $30 \mathrm{c}$ treatments were analyzed by liquid chromatography-mass spectrometry (LC-MS), the left panel is the cholesterol standard curve, and the right panel is the calculated results. (C) The concentrations of endogenous PC, PE, and SM in cell supernatant after M $\beta C D$ and 30 treatments were analyzed by LC-MS, from top to bottom are the concentration changes of PC, PE, and SM. Significant differences were analyzed by a two-way ANOVA test. Scale bar $=20 \mu \mathrm{m}$. Henceforth, ${ }^{*},{ }^{* \star},{ }^{* \star *}$, and ${ }^{* * * \star}, P<0.05,0.01,0.001$, and 0.0001, respectively. 

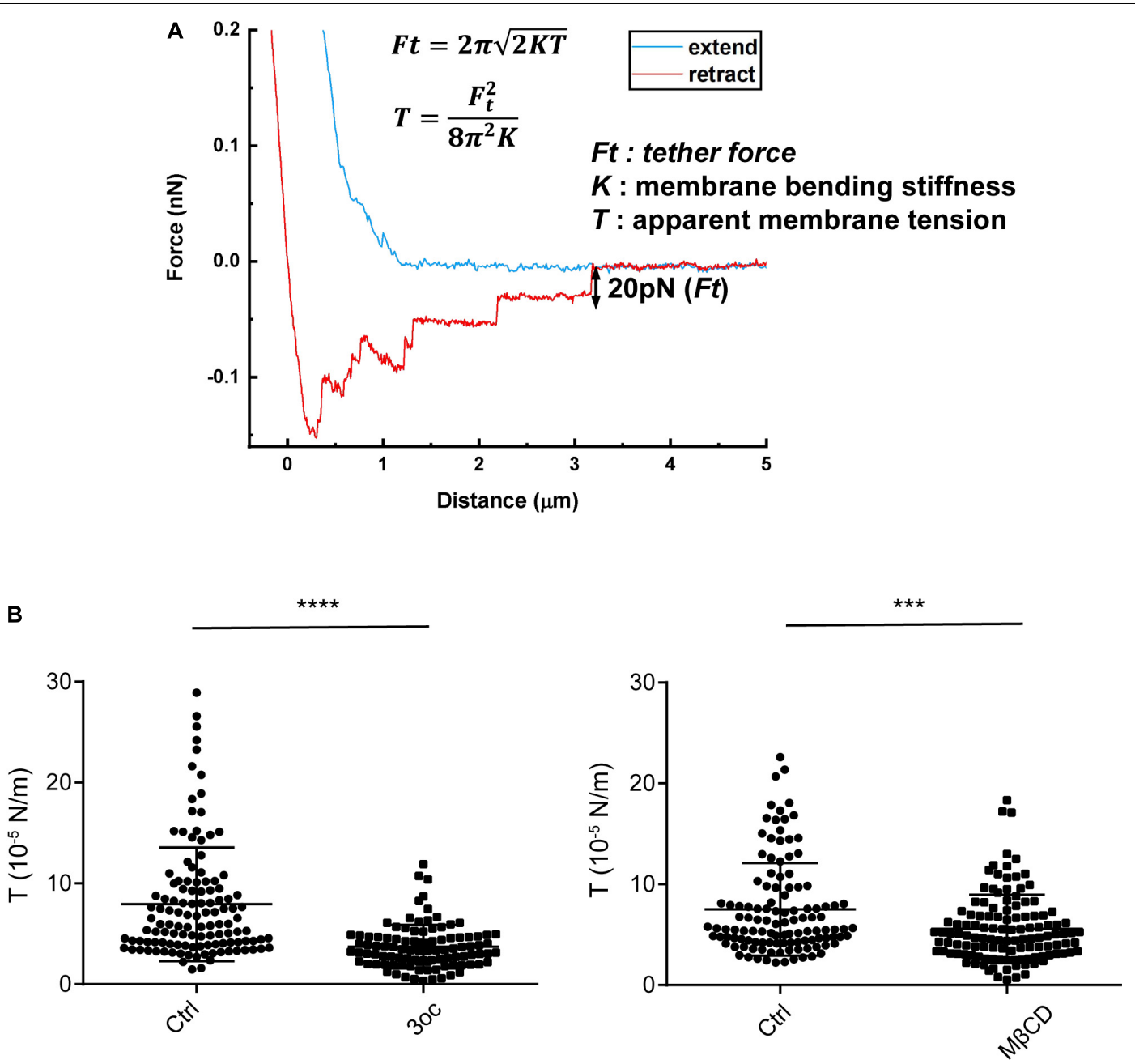

FIGURE 3 | Membrane tension probed by atomic force microscopy (AFM)-based tether force measurements. (A) A typical force curve showing membrane tethers was pulled out from the cell membrane and broken in a stepwise manner. The last step size (i.e., tether force, Ft) was used to compute apparent membrane tension (T), based on the formula (inset), where $K$ is membrane bending stiffness and is about $3 \times 10^{-19} \mathrm{~N} / \mathrm{m}$ on average. (B) Membrane tension of Hela cells in resting state and after the treatment of $30 \mathrm{c}$ and $\mathrm{M} \beta \mathrm{CD}$, respectively. For $30 \mathrm{c}$ and $\mathrm{M} \beta \mathrm{CD}$ experiments, force probing was started after the 15-min incubation. Each data point was from one force measurement, and for each cell, 3 measurements were performed and about 50 cells were probed for each experimental condition. ${ }^{\star \star \star \star} P<0.0001$ and ${ }^{\star \star *} P<0.0003$ as measured by an unpaired $t$-test.

analyzed EGFR phosphorylation at tyrosine 1,173 in the absence of its ligand (Chen and Resh, 2002). Clearly, both treatments led to a substantial increase of pEGFR, suggesting in this assay the two reagents are functionally equivalent (Figure 4A). However, the peak phosphorylation of 3oc treatment took place $30 \mathrm{~min}$ earlier than $\mathrm{M} \beta \mathrm{CD}$ treatment. Similarly, lipid domain disruption causes spontaneous ERK activation (Kabouridis et al., 2000). Figure 4B shows that both $30 \mathrm{oc}$ and $\mathrm{M} \beta \mathrm{CD}$ led to a similar extent of ERK phosphorylation. Caveolae are caveolin-dependent, specialized lipid rafts characterized by an inward indented membrane "cave" and an abundance of PIP2 in the corresponding inner leaflets (Fujita et al., 2009). They are a significant source of membrane endocytosis. We first treated THP-1 cells with CPZ, which blocks clathrin-dependent internalization, and then with LPS to trigger caveolae-dependent TLR4 receptor endocytosis (Varkevisser et al., 2013). Flow cytometry results showed that the average fluorescence intensity of TLR4 on the cell membrane increased after treatment, while $\mathrm{CPZ}$ treatment inhibited the internalization from $674.3 \pm 7.9$ to $848 \pm 9.6$, both $\mathrm{M} \beta \mathrm{CD}$ and $30 c$ treatments further increased the membrane retention of TLR4 from $848 \pm 9.6$ to $945.7 \pm 20$ and $913.3 \pm 9.3$, respectively, suggesting the effective blockage of caveolae function by both treatments (Figure 4C).

\section{A Low Concentration of 3-Oxododecanoyl Is a Generalizable Raft Modifier Without Altering the Cell Survival}

Unlike non-myeloid cells, immune cells are sensitive to 3ocinduced apoptosis (Song et al., 2019), we wondered if $30 \mathrm{c}$ treatment on THP-1 cells can be precisely controlled to permit 
A

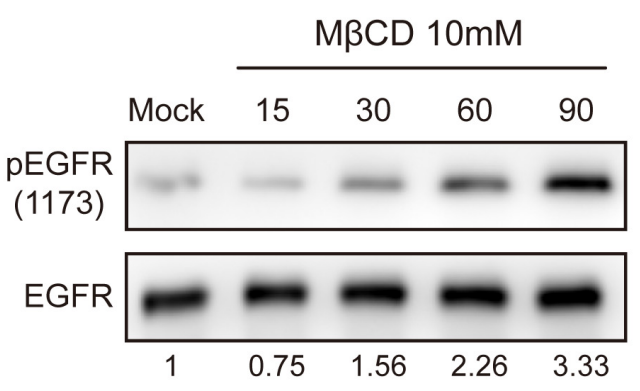

B
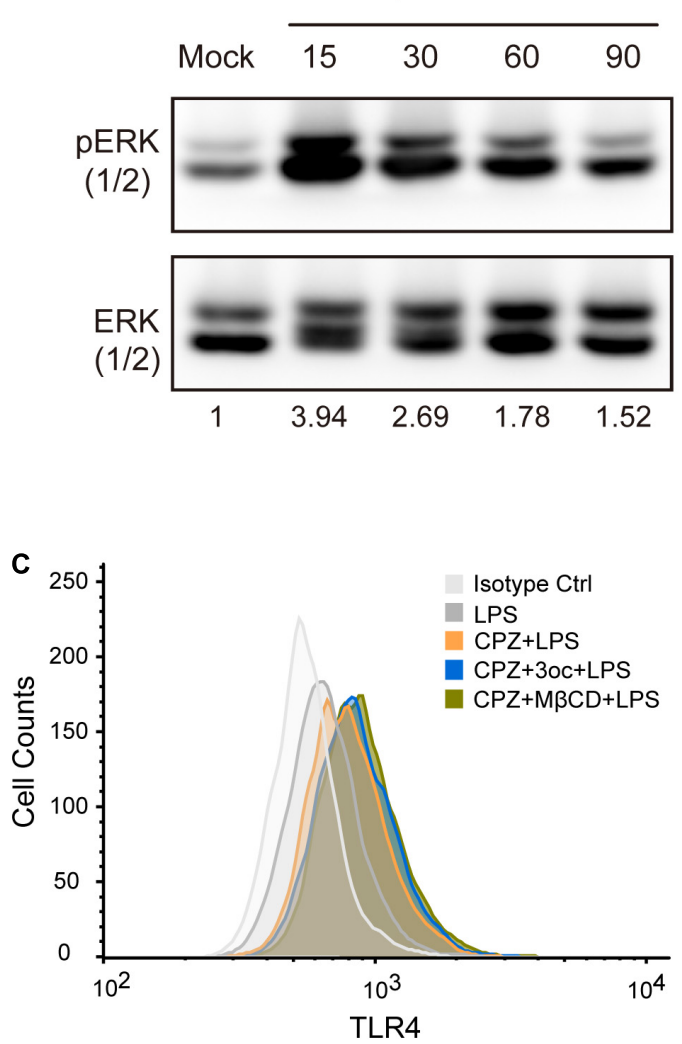

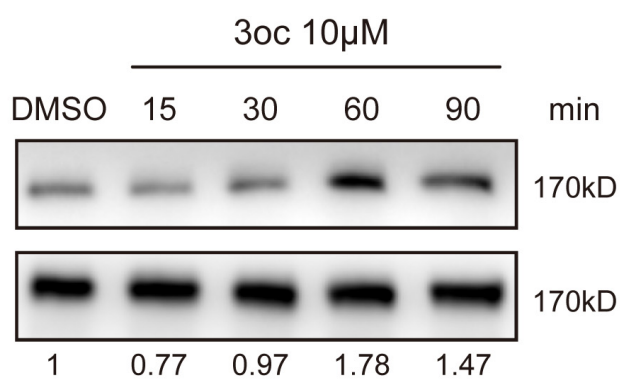

$30 \mathrm{oc} 10 \mu \mathrm{M}$
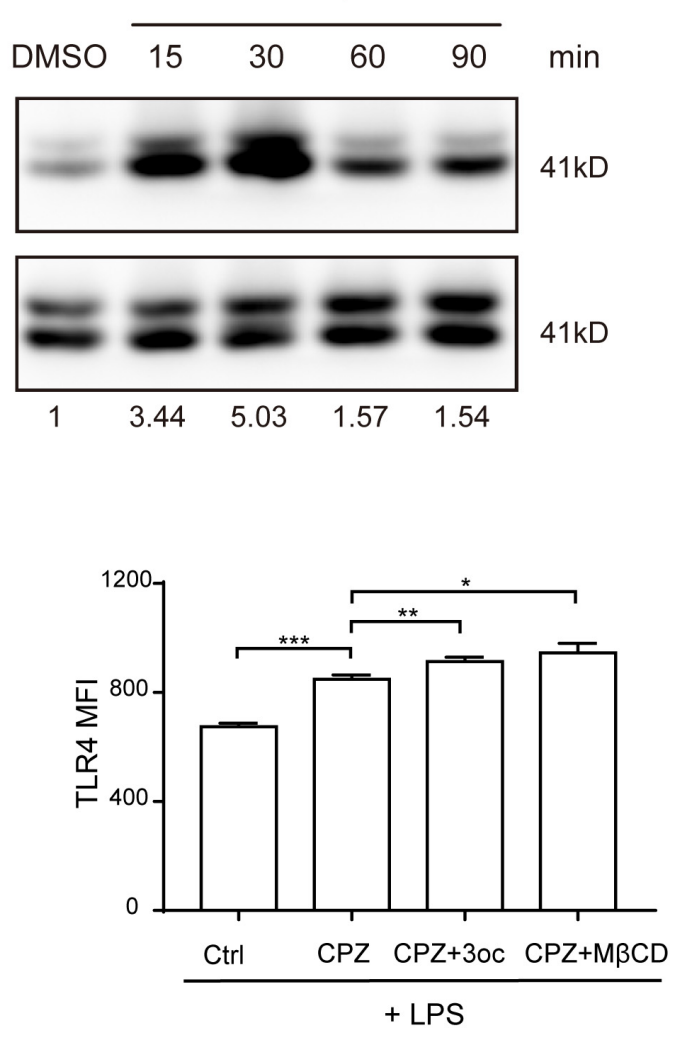

FIGURE 4 | The effects of lipid-ordered domain disruption on cell signal transduction. (A) M $\beta C D$ and 3oc treatments induce spontaneous phosphorylation of epidermal growth factor receptor (EGFR) at tyrosine 1,173 at the indicated time. (B) Lipid-ordered domain disruption leads to a similar extent of extracellular signal-regulated kinase (ERK) phosphorylation. (C) The disruption of the lipid-ordered domain leads to the obstruction of lipid raft-dependent endocytosis of TLR4, the left panel is the representative histogram of the flow cytometry expression of TLR 4 on THP- 1 cells treated with LPS (10 $\mu \mathrm{g} / \mathrm{ml})$, LPS + CPZ, LPS + CPZ $+30 c$, or $\mathrm{LPS}+\mathrm{CPZ}+\mathrm{M} \beta \mathrm{CD}$, and the right panel is the quantitative data shown as mean fluorescence intensity $\pm \mathrm{SEM}$. The values reported under each blot are densitometry analysis relative to controls taken as 1 after normalization by internal reference. All experiments were repeated three times.

lipid domain analysis without triggering significant cell death. As the $30 c$ concentration used in this report was maintained at $10 \mu \mathrm{M}$, we performed a dose-response analysis. In this study, 10 and $20 \mu \mathrm{M}$ of $30 c$ did not significantly alter the cell survival, while higher concentrations led to measurable apoptosis at $100 \mu \mathrm{M}$ (Figure $\mathbf{5 A}$, and the results of the flow cytometry analysis were shown in Supplementary Figure 6). At the higher concentrations, THP-1 cell death was mediated by the canonical Caspase 3 pathway, leading to its cleavage after about $30 \mathrm{~min}$ treatment with $30 \mathrm{c}$ at $30 \mu \mathrm{M}$ (Figure 5B).

\section{DISCUSSION}

Compared with $\mathrm{M} \beta \mathrm{CD}$ with its working mechanism proposed decades ago, we currently do not understand how 3oc works. In our previous study (Song et al., 2018), we showed that to disrupt lipid rafts, the acyl chains of homoserine lactones must be no shorter than 10 carbons and require a doublebonded oxygen following the homoserine. The acyl chain length was as expected to correlate with their ability of membrane insertion. The oxo group, in contrast, may 

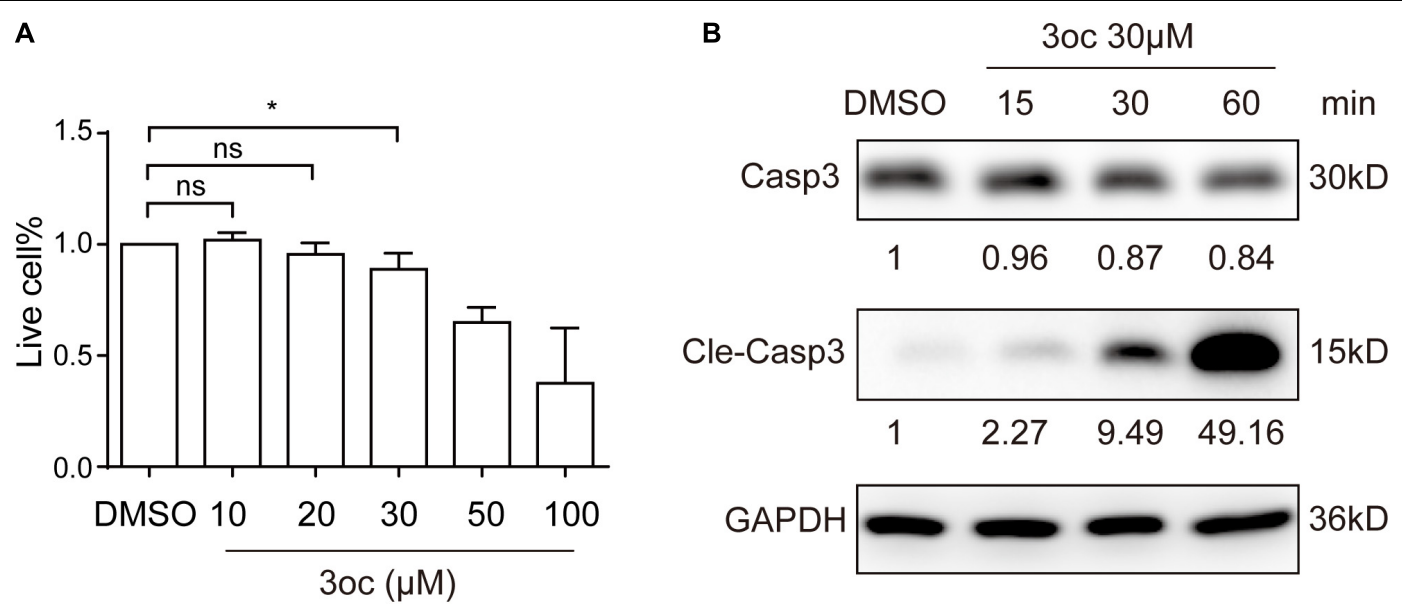

FIGURE 5 | Cell viability of THP-1 cells treated with different concentrations of 3oc. (A) Cell apoptosis was detected by the Annexin V/PI apoptosis detection kit and then measured by flow cytometry, statistical analysis results of three experiments. Live cell\% = (Annexin $\mathrm{V}$ negative cells in M $\beta C D$ or $30 \mathrm{c}$ treatment/total cells)/(Annexin $V$ negative cells in DMSO treatment/total cells) $\times 100 \%$. (B) The Western blot results show that when the $30 c$ concentration reaches $30 \mu M$, and it can induce obvious activation of Caspase 3. The values reported under each blot are densitometry analysis relative to controls taken as 1 after normalization by internal reference. All experiments were repeated three times, and the bars represent the mean $\pm \mathrm{SD}(n=3) ;{ }^{*} P<0.046$ as measured by an unpaired $t$-test.

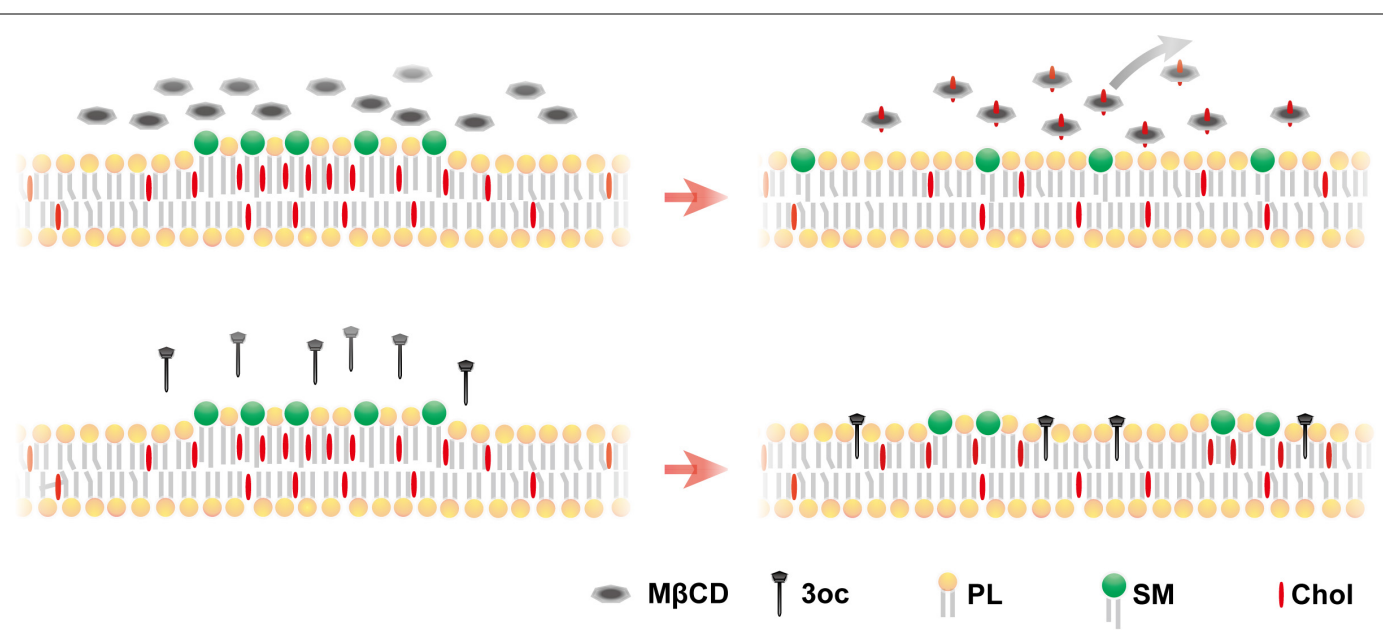

FIGURE 6 | Proposed mechanism for M $\beta C D$ and 3oc disruption of the lipid-ordered domain. Upper panel: M $\beta C D$ treatment produces a more homogeneous plasma membrane due to the extraction of lipid components. Lower panel: 3oc produces a more limited disintegration with small islands remaining, without affecting the plasma membrane components [(phospholipids (PLs)].

be the key to cause the breaking of the semicrystalline structure formed between gangliosides and cholesterol (Simons and Toomre, 2000).

Our results suggest that $3 o c$ is a viable replacement for $\mathrm{M} \beta \mathrm{CD}$ for the purpose of lipid-ordered domain disruption. The $3 \mathrm{oc}$ is to some extent more efficient and requires lower effective concentrations, and in some systems, the effect is more rapid. Perhaps the most advantageous feature is the retention of all classes of membrane lipids after the treatment, while $\mathrm{M} \beta \mathrm{CD}$ leads to a dramatic reduction in all (Figure 6). The non-specific depletion by $\mathrm{M} \beta \mathrm{CD}$ was reported previously, and it was considered as a consequence of high M $B C D$ (Sanchez et al., 2011). Our results suggest that this off-target effect is measurable in common concentrations. 3oc is therefore expected to impact cell biology to a lesser extent. As the studies of lipidordered domain almost inevitably use $\mathrm{M} \beta \mathrm{CD}$ as the de novo treatment, it is possible that some of the effects, particularly those related to receptor signaling on internalization, such as CD95 and insulin receptor (Di Guglielmo et al., 1998; Lee et al., 2006), may not be entirely attributable to lipid domain disruption. Whether $30 c$ offers a distinct advantage with reduced membrane replenishment therein remains to be fully investigated.

A note of caution is that in some cell types, particularly immune cells, 3oc treatment may induce TNFR1-dependent cell death at high concentrations, which may require an evaluation of the dosage used. In our laboratory, the blockage of Caspase 8 or deficiency of TNFR1 inhibited cell death, which may serve as a reference if $30 c$ is used on immune cells. 


\section{DATA AVAILABILITY STATEMENT}

The original contributions presented in the study are included in the article/Supplementary Material, further inquiries can be directed to the corresponding author/s.

\section{AUTHOR CONTRIBUTIONS}

HR, CZ, and YX performed all the experiments. XF, TX, and YS conceptualized and designed the experiments. YS wrote the manuscript. All authors contributed to the article and approved the submitted version.

\section{FUNDING}

YS was supported by the Joint Peking-Tsinghua Center for Life Sciences, the National Natural Science Foundation of China General Program (31370878), the State Key Program (31630023), and the Innovative Research Group Program (81621002), by grants from CIHR (PJT-156334 and PJT-166155) and NSERC

\section{REFERENCES}

Asakura, K., Ueda, A., and Mutoh, T. (2015). Lipid rafts and their possible involvements in neuroimmunological disorders. Front. Biosci. 20, 303-313. doi: $10.2741 / 4310$

Bagatolli, L. A. (2006). To see or not to see: lateral organization of biological membranes and fluorescence microscopy. Biochim. Biophys. Acta 1758, 15411556. doi: 10.1016/j.bbamem.2006.05.019

Baumgart, T., Hammond, A. T., Sengupta, P., Hess, S. T., Holowka, D. A., Baird, B. A., et al. (2007). Large-scale fluid/fluid phase separation of proteins and lipids in giant plasma membrane vesicles. Proc. Natl. Acad. Sci. US A. 104, 3165-3170. doi: 10.1073/pnas.0611357104

Casares, D., Escribá, P. V., and Rosselló, C. A. (2019). Membrane Lipid Composition: effect on Membrane and Organelle Structure, Function and Compartmentalization and Therapeutic Avenues. Int. J. Mol. Sci. 20:2167.

Chen, X., and Resh, M. D. (2002). Cholesterol depletion from the plasma membrane triggers ligand-independent activation of the epidermal growth factor receptor. J. Biol. Chem. 277, 49631-49637. doi: 10.1074/jbc.M2083 27200

Di Guglielmo, G. M., Drake, P. G., Baass, P. C., Authier, F., Posner, B. I., and Bergeron, J. J. (1998). Insulin receptor internalization and signalling. Mol. Cell. Biochem. 182, 59-63. doi: 10.1007/978-1-4615-5647-3_6

Fujita, A., Cheng, J., Tauchi-Sato, K., Takenawa, T., Fujimoto, T., and distinct, A. (2009). pool of phosphatidylinositol 4,5-bisphosphate in caveolae revealed by a nanoscale labeling technique. Proc. Natl. Acad. Sci. U S A. 106, 9256-9261. doi: 10.1073/pnas.0900216106

García-Arribas, A. B., Alonso, A., and Goñi, F. M. (2016). Cholesterol interactions with ceramide and sphingomyelin. Chem. Phys. Lipids 199, 26-34.

Kabouridis, P. S., Janzen, J., Magee, A. L., and Ley, S. C. (2000). Cholesterol depletion disrupts lipid rafts and modulates the activity of multiple signaling pathways in T lymphocytes. Eur. J. Immunol. 30, 954-963. doi: 10.1002/15214141(200003)30:3\&lt;954::AID-IMMU954\&gt;3.0.CO;2-Y

Kapus, A., and Janmey, P. (2013). Plasma membrane-cortical cytoskeleton interactions: a cell biology approach with biophysical considerations. Compr. Physiol. 3, 1231-1281. doi: 10.1002/cphy.c120015

Kusumi, A., Nakada, C., Ritchie, K., Murase, K., Suzuki, K., Murakoshi, H., et al. (2005). Paradigm shift of the plasma membrane concept from the two-dimensional continuum fluid to the partitioned fluid: high-speed singlemolecule tracking of membrane molecules. Annu. Rev. Biophys. Biomol. Struct. 34, 351-378. doi: 10.1146/annurev.biophys.34.040204.144637
(RGPIN/03748-2018). XF was supported by the National Natural Science Foundation of China (21735006, 22077124, and 91939301). HR was supported by the National Natural Science Foundation of China (32000873) and the China Postdoctoral Science Foundation (2019M650718).

\section{ACKNOWLEDGMENTS}

We would like to thank Weihua Wang (Center of Pharmaceutical Technology, Tsinghua University) for helping with LCMS experiments and Ran Du (Metabolomics Platform at Agricultural Genomics Institute at Shenzhen, Chinese Academy of Agricultural Sciences) for providing the AB SCIEX QTRAP 5500 system.

\section{SUPPLEMENTARY MATERIAL}

The Supplementary Material for this article can be found online at: https://www.frontiersin.org/articles/10.3389/fphys. 2021.758458/full\#supplementary-material

Lee, K. H., Feig, C., Tchikov, V., Schickel, R., Hallas, C., Schütze, S., et al. (2006) The role of receptor internalization in CD95 signaling. EMBO J. 25, 1009-1023. doi: 10.1038/sj.emboj.7601016

Lee, M. Y., Ryu, J. M., Lee, S. H., Park, J. H., and Han, H. J. (2010). Lipid rafts play an important role for maintenance of embryonic stem cell self-renewal. J. Lipid Res. 51, 2082-2089. doi: 10.1194/jlr.M001545

Levental, I., Levental, K. R., and Heberle, F. A. (2020). Lipid Rafts: controversies Resolved. Trends Cell. Biol. 30, 341-353. doi: 10.1016/j.tcb.2020.01.009

Levental, I., Lingwood, D., Grzybek, M., Coskun, U., and Simons, K. (2010). Palmitoylation regulates raft affinity for the majority of integral raft proteins. Proc. Natl. Acad. Sci. U S A. 107, 22050-22054. doi: 10.1073/pnas.1016184107

Li, J., Wijeratne, S. S., Nelson, T. E., Lin, T. C., He, X., Feng, X., et al. (2020). Dependence of Membrane Tether Strength on Substrate Rigidity Probed by Single-Cell Force Spectroscopy. J. Phys. Chem. Lett. 11, 4173-4178. doi: 10. 1021/acs.jpclett.0c00730

López, C. A., de Vries, A. H., and Marrink, S. J. (2011). Molecular mechanism of cyclodextrin mediated cholesterol extraction. PLoS Comput. Biol. 7:e1002020. doi: 10.1371/journal.pcbi.1002020

Mu, L., Tu, Z., Miao, L., Ruan, H., Kang, N., Hei, Y., et al. (2018). A phosphatidylinositol 4,5-bisphosphate redistribution-based sensing mechanism initiates a phagocytosis programing. Nat. Commun. 9:4259. doi: 10.1038/s41467-018-06744-7

Nakayama, H., Nagafuku, M., Suzuki, A., Iwabuchi, K., and Inokuchi, J. I. (2018). The regulatory roles of glycosphingolipid-enriched lipid rafts in immune systems. FEBS Lett. 592, 3921-3942. doi: 10.1002/1873-3468.13275

Ohvo, H., and Slotte, J. P. (1996). Cyclodextrin-mediated removal of sterols from monolayers: effects of sterol structure and phospholipids on desorption rate. Biochemistry 35, 8018-8024. doi: 10.1021/bi9528816

Orbach, R., and Su, X. (2020). Surfing on Membrane Waves: microvilli, Curved Membranes, and Immune Signaling. Front. Immunol. 11:2187. doi: 10.3389/ fimmu.2020.02187

Pike, L. J. (2006). Rafts defined: a report on the Keystone Symposium on Lipid Rafts and Cell Function. J. Lipid Res. 47, 1597-1598. doi: 10.1194/jlr.E600002-JLR200

Pontes, B., Monzo, P., and Gauthier, N. C. (2017). Membrane tension: a challenging but universal physical parameter in cell biology. Semin. Cell Develop. Biol. 71, 30-41.

Pralle, A., Keller, P., Florin, E. L., Simons, K., and Hörber, J. K. (2000). Sphingolipid-cholesterol rafts diffuse as small entities in the plasma membrane of mammalian cells. J. Cell Biol. 148, 997-1008. doi: 10.1083/jcb.148.5. 997 
Sanchez, S. A., Gunther, G., Tricerri, M. A., and Gratton, E. (2011). Methyl$\beta$-cyclodextrins preferentially remove cholesterol from the liquid disordered phase in giant unilamellar vesicles. J. Membr. Biol. 241, 1-10. doi: 10.1007/ s00232-011-9348-8

Sheetz, M. P. (2001). Cell control by membrane-cytoskeleton adhesion. Nat. Rev. Mol. Cell Biol. 2, 392-396. doi: 10.1038/35073095

Shi, Y., and Ruan, H. (2020). Toward a Membrane-Centric Biology. Front. Immunol. 11:1909. doi: 10.3389/fimmu.2020.01909

Simons, K., and Toomre, D. (2000). Lipid rafts and signal transduction. Nat. Rev. Mol. Cell Biol. 1, 31-39.

Song, D., Meng, J., Cheng, J., Fan, Z., Chen, P., Ruan, H., et al. (2018). Pseudomonas aeruginosa quorum-sensing metabolite induces host immune cell death through cell surface lipid domain dissolution. Nat. Microbiol. 4, 97-111. doi: 10.1038/s41564-018-0290-8

Song, D., Meng, J., Cheng, J., Fan, Z., Chen, P., Ruan, H., et al. (2019). Pseudomonas aeruginosa quorum-sensing metabolite induces host immune cell death through cell surface lipid domain dissolution. Nat. Microbiol. 4, 97-111. doi: 10.1038/s41564-018-0290-8

Sun, M., Graham, J. S., Hegedus, B., Marga, F., Zhang, Y., Forgacs, G., et al. (2005). Multiple membrane tethers probed by atomic force microscopy. Biophys. J. 89, 4320-4329. doi: 10.1529/biophysj.104.058180

Varkevisser, R., Houtman, M. J. C., Waasdorp, M., Man, J. C. K., Heukers, R., Takanari, H., et al. (2013). Inhibiting the clathrin-mediated endocytosis pathway rescues K(IR)2.1 downregulation by pentamidine. Pflugers Archiv-European. J. Physiol. 465, 247-259. doi: 10.1007/s00424-012-11 89-5
Vona, R., Iessi, E., and Matarrese, P. (2021). Role of Cholesterol and Lipid Rafts in Cancer Signaling: a Promising Therapeutic Opportunity? Front. Cell Dev. Biol. 9:622908. doi: 10.3389/fcell.2021.622908

Wang, C., Yu, Y., and Regen, S. L. (2017). Lipid Raft Formation: key Role of Polyunsaturated Phospholipids. Angew. Chem. Int. Ed. 56, 1639-1642. doi: 10.1002/anie.201611367

Zhang, T., Hu, W., and Chen, W. (2021). Plasma Membrane Integrates Biophysical and Biochemical Regulation to Trigger Immune Receptor Functions. Front. Immunol. 12:613185. doi: 10.3389/fimmu.2021.613185

Conflict of Interest: The authors declare that the research was conducted in the absence of any commercial or financial relationships that could be construed as a potential conflict of interest.

Publisher's Note: All claims expressed in this article are solely those of the authors and do not necessarily represent those of their affiliated organizations, or those of the publisher, the editors and the reviewers. Any product that may be evaluated in this article, or claim that may be made by its manufacturer, is not guaranteed or endorsed by the publisher.

Copyright $\odot 2022$ Ruan, Zou, Xu, Fang, Xia and Shi. This is an open-access article distributed under the terms of the Creative Commons Attribution License (CC BY). The use, distribution or reproduction in other forums is permitted, provided the original author(s) and the copyright owner(s) are credited and that the original publication in this journal is cited, in accordance with accepted academic practice. No use, distribution or reproduction is permitted which does not comply with these terms. 